\title{
SPONTANEOUS RELIEVING RETINOTOMIES IN DIABETIC SILICONE FILLED EYES
}

\author{
N. WILSON-HOLT and Z. GREGOR \\ London
}

\begin{abstract}
SUMMARY
We describe in six patients the delayed occurrence of inferior tractional retinal detachments following epiretinal reproliferation 4 to 5 months after vitrectomy and silicone oil tamponade for diabetic related retinal detachments. The epiretinal traction was spontaneously relieved by the occurrence of large inferior tension breaks which subsequently enlarged and coalesced to form retinotomies.

This process appears to be relatively benign as it remains limited to the peripheral retina, and in all six patients the posterior retina has remained attached for a minimum of 12 months follow up, with improved central visual acuity.
\end{abstract}

Neovascularisation in proliferative diabetic retinopathy is characteristically confined to the posterior retina. However, fibrovascular proliferation or postoperative reproliferation may occur well anterior to the equator and extend to the ora serrata; when associated with an extensive retinal detachment, surgical management of such anterior membranes may prove difficult.

The treatment of associated retinal detachments by vitrectomy and epiretinal membrane dissection is an established method with reproducibly good results. ' ${ }^{4}$ The usefulness of silicone oil as a tamponading agent in the management of particularly complicated retinal detachments in diabetic eyes has been documented. ${ }^{56}$ Its use allows for early postoperative photocoagulation, prolonged closure of retinal breaks and for confinement of postoperative haemorrhage. However silicone oil may result in anterior segment complications such as cataract, glaucoma and keratopathy. ${ }^{7-9}$ In the posterior segment its major disadvantage is late reproliferation of epiretinal membranes and subsequent retinal redetachment with or without associated retinal breaks. ${ }^{1011}$ The latter can be difficult to manage and may preclude the removal of silicone oil at that stage.

The purpose of this paper is to describe the delayed occurrence of tractional retinal detachments associated

Correspondence to: Mr. N. Wilson-Holt FRCS, Moorfields Eye Hospital, City Road, London ECIV 2PD, UK. with enlarged tension tears following epiretinal reproliferation after vitrectomy and silicone oil tamponade for diabetic retinal detachments.

The epiretinal traction was relieved by the occurrence of inferior breaks which subsequently enlarged and coalesced to form spontaneous relieving retinotomies. This process appears to be relatively benign as it remains limited to the peripheral retina, permitting the posterior retina to remain attached.

\section{MATERIALS AND METHODS}

We reviewed six patients who underwent closed intraocular microsurgery with silicone oil tamponade for tractional or combined rhegmatogenous/tractional retinal detachment associated with advanced proliferative diabetic retinopathy. The operations took place at Moorfields Eye Hospital between June 1989 and January 1991. Three patients were male, three female. The age range was 28-62 years, with a mean of 38.3 years (Table I). Five eyes in five patients underwent primary vitrectomy, delamination of epiretinal membranes, fluid/air exchange when indicated, peroperative endolaser photocoagulation, and silicone oil tamponade. One patient had undergone previous vitrectomy, delamination, fluid/ $\mathrm{SF}_{6}$ gas exchange, but the retina had redetached, so necessitating further surgery.

\section{RESULTS}

In the six patients we observed, the duration of traction retinal detachment prior to surgery was between six and nine months. The onset of reproliferation ranged between two and four months with a mean of 3.2 months. The commonest site of reproliferation was the inferior retina at six o'clock.

In all six patients tension tears inferiorly were noted at four months, with development of inferior retinal detachment anterior to the equator (Fig. 1) and associated with subretinal silicone oil between four and five months following surgery (Figs. 2 and 3 ).

Extension and coalescence of tension tears to form a spontaneous relieving retinotomy (Fig. 4) occurred 
Table I. Patient data

\begin{tabular}{|c|c|c|c|c|c|c|}
\hline Patient & Age & Pre-op VA & $\begin{array}{l}\text { Time to develop } \\
\text { tension tears }\end{array}$ & $\begin{array}{l}\text { Time to develop } \\
\text { retinotomy }\end{array}$ & $\begin{array}{c}\text { Follow } \\
\text { up (mths) }\end{array}$ & $\begin{array}{l}\mathrm{VA} \\
\text { at FU }\end{array}$ \\
\hline $1 \mathrm{M}$ & 62 & $\mathrm{CF}$ & 4 months & 5.5 months & 12 & $6 / 36$ \\
\hline $3 \mathrm{M}$ & 28 & $\mathrm{CF}$ & 4 months & 7 months & 18 & $6 / 24$ \\
\hline $4 \mathrm{~F}$ & 38 & $\mathrm{CF}$ & 1.5 months & 4.5 months & 11 & $6 / 36$ \\
\hline $5 \mathrm{M}$ & 29 & HM & 2.5 months & 5 months & 15 & $6 / 60$ \\
\hline $6 \mathrm{~F}$ & 41 & $\mathrm{HM}$ & 3 months & 6 months & 11 & $\mathrm{CF}$ \\
\hline
\end{tabular}

between five and seven months (mean 5.8 months), and in all six patients the retina posterior to the equator has remained attached over a minimum of 12 months follow up.

\section{ILLUSTRATIVE CASE REPORTS}

\section{Case 1}

A 62 year old male diabetic was referred in December 1990 for surgical management of combined tractional and rhegmatogenous retinal detachment in the right eye. Visual acuities at that stage were counting fingers in the right eye and 6/60 in the left eye. Because of moderately dense cataract, extracapsular cataract extraction was performed, together with vitrectomy and delamination of epiretinal membranes well anterior to the equator. At surgery, a large break was noted in the superotemporal periphery, and fluid/air exchange followed by endolaser retinopexy and then air/silicone oil exchange performed.

Immediately after surgery, the retina was reattached, but over the ensuing four months an inferior tractional retinal detachment developed followed by tension breaks and the entry of silicone oil into the subretinal space. These breaks coalesced to form a large spontaneous retinotomy,

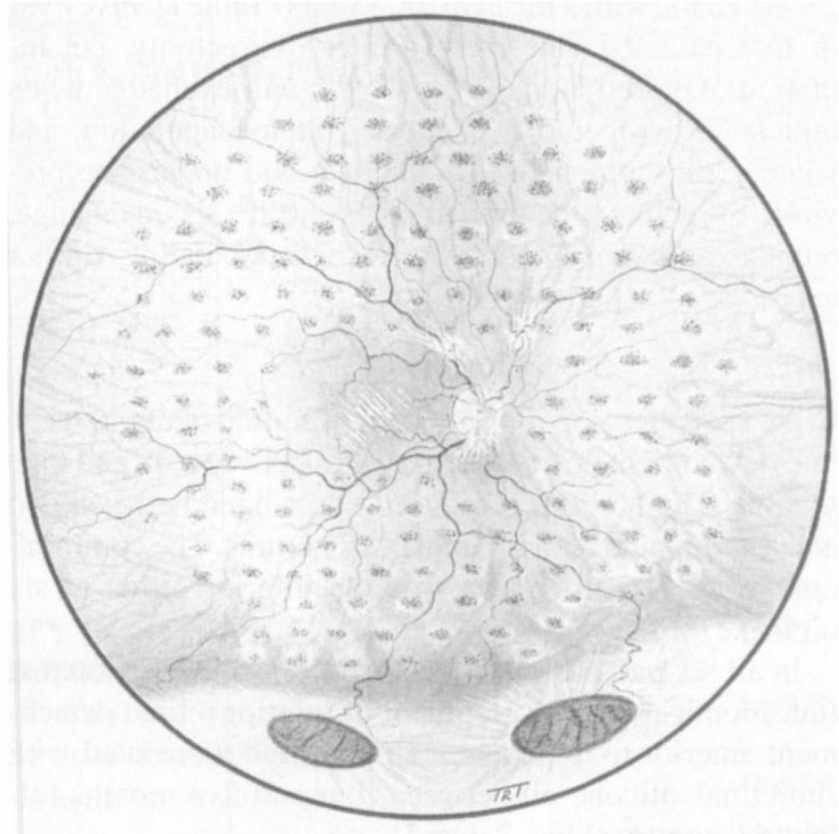

Fig. 1. Fundus drawing depicting development of inferior peripheral retinal traction detachment and oval tension tears in a diabetic silicone oil filled eve following vitrectomy, delamination of epiretinal membranes and panretinal photocoagulation. and the subretinal oil appeared to join the main oil globule in the vitreous cavity.

The patient has been followed up for eight months, and the retina has remained stable with a small localised globule of subretinal oil in the inferotemporal periphery, but the posterior retina attached, and visual acuity remains $6 / 36$.

\section{Case 2}

A 32 year old female diabetic was referred in October 1989 for surgical management of tractional retinal detachment in the right eye. Visual acuities at presentation were counting fingers in the right eye and $6 / 6$ in the left eye. In the right eye there was extensive fibrovascular proliferation, with a 'table top' tractional retinal detachment involving the macula. Vitrectomy and delamination of epiretinal membranes were performed, but no retinal break was found at the time of surgery. Endolaser panretinal phototocoagulation was applied where possible, and in view of the extensive detachment and retinal ischaemia fluid/silicone oil exchange performed. The retina remained attached for six weeks, but thereafter marked reproliferation of fibrovascular tissue was noted nasal to the optic disc associated with a tractional retinal detachment involving the macula. Further delamination and segmentation of epiretinal membranes posterior to the equator was performed, but reproliferation occurred again involving the anterior retina inferotemporally. Seven weeks later, a large inferior tension tear was noted and this was associated with subretinal silicone oil. Further tension tears then developed adjacent to the original break and these subsequently coalesced to form a spontaneous retinotomy.

The retina has remained stable over 18 months follow up, with a small inferotemporal pool of subretinal silicone oil, but the retina otherwise remaining attached, visual acuity being $6 / 24$.

\section{Case 3}

A 28 year old male diabetic was referred for vitrectomy to the right eye in June 1989. In spite of extensive panretinal photocoagulation to both eyes, visual acuities at that stage were counting fingers in the right eye and light perception in the left eye. Fundal examination of the right eye showed a very active posterior fibrovascular proliferation associated with a combined tractional and rhegmatogenous retinal detachment involving the macula. The left eye was considered inoperable. 


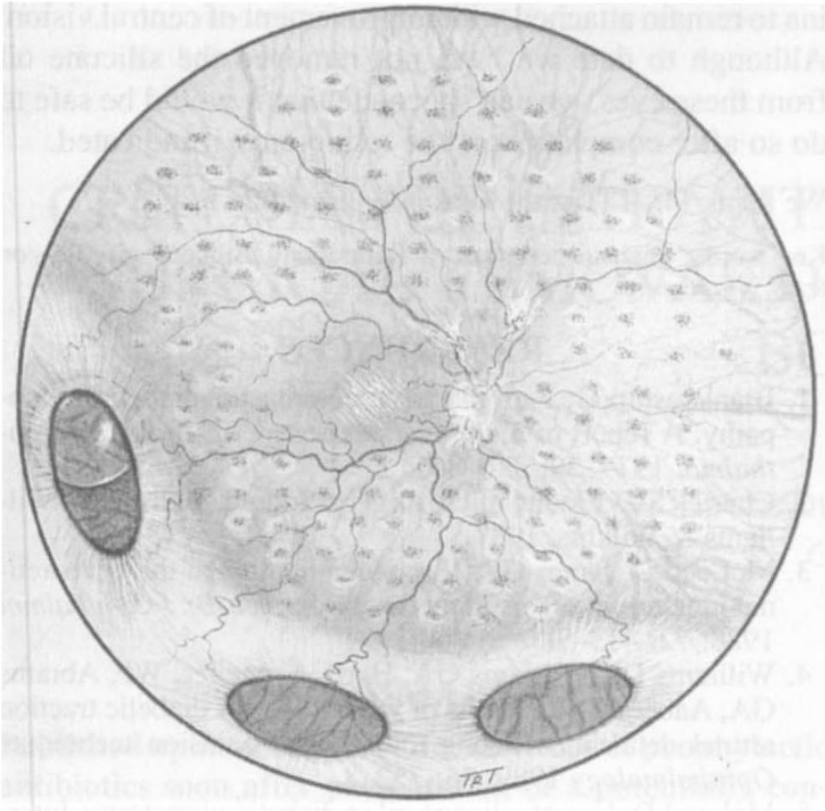

Fig 2.

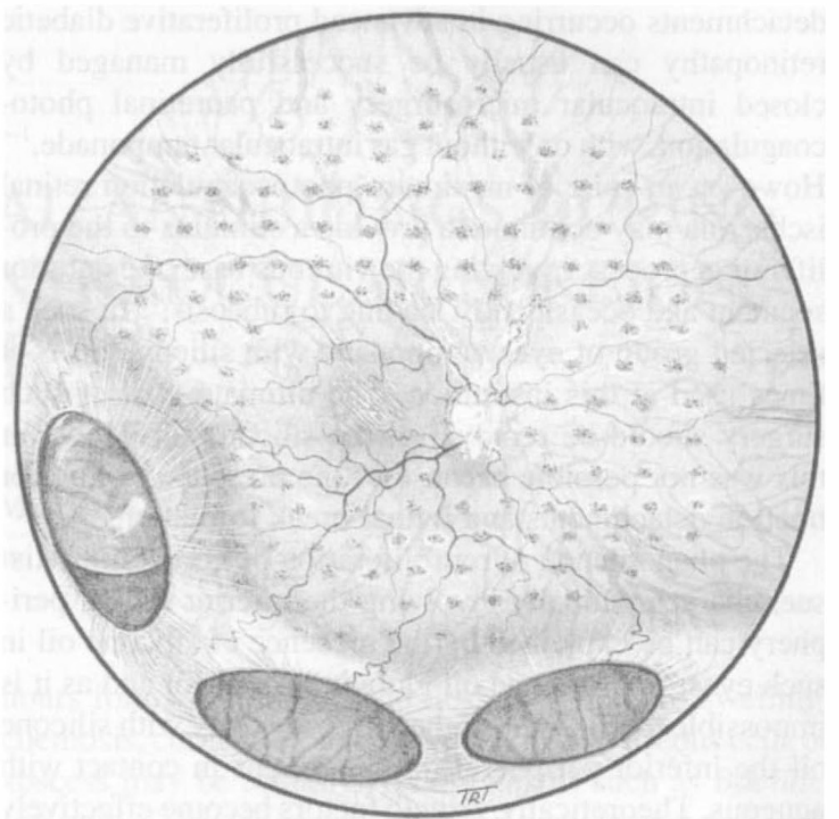

Fig 3.

Figs. 2 and 3. Progression of the peripheral traction retinal detachment, enlargement of existing tears, and development of a further retinal tear under tension, with occurrence of subretinal silicone oil.

Following extensive delamination of epiretinal membranes, cryotherapy and fluid $30 \%$ sulphur hexafluoride exchange, the retina attached initially but redetached two months later. At the time of reoperation marked anterior proliferation was found. Following lensectomy the fibrovascular tissue was successfully removed and fluid/silicone oil exchange together with encirclement with a 240 band performed. Over two months further anterior proliferation occurred and an anterior retinal break developed leading to a peripheral retinal detachment. This was

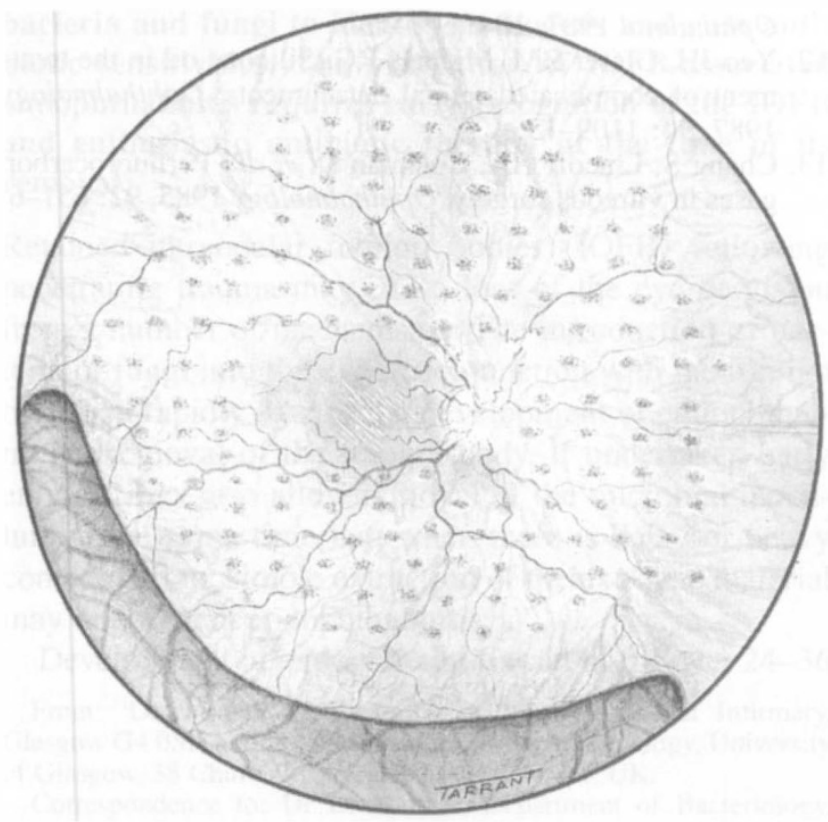

Fig. 4. Extension and coalescence of tension tears to form a spontaneous relieving retinotomy. treated by a top-up of silicone oil and endolaser photocoagulation, but six weeks thereafter a small inferior retinal detachment was noted with subretinal oil present and a single inferior peripheral retinal tension tear. At this stage, the retina was otherwise attached and vision had improved to $6 / 24$.

Over the ensuing three months, further peripheral tension tears developed, associated with peripheral retinal detachment and subretinal silicone oil. The breaks gradually enlarged and coalesced to form a spontaneous inferior retinotomy. Vision has remained stable at $6 / 24$ over 18 months follow up.

\section{Case 4}

A 38 year old female insulin dependent diabetic was referred in January 1991 for surgical management of combined tractional and rhegmatogenous retinal detachment in her right eye. Visual acuity at the time of presentation was counting fingers in the right eye and 6/36 in the left eye.

Vitrectomy, delamination of epiretinal membranes and fluid/silicone oil exchange was performed in February 1991. Postoperatively the retina remained attached for six weeks when an inferior retinal detachment associated with an inferior tension tear and subretinal silicone oil was noted.

Over the ensuing three months further inferior breaks occurred leading to the development of a spontaneous retinotomy. Visual acuity at six months follow up has remained stable at $6 / 36$.

\section{DISCUSSION}

Tractional or combined tractional/rhegmatogenous retinal 
detachments occurring in advanced proliferative diabetic retinopathy can usually be successfully managed by closed intraocular microsurgery and panretinal photocoagulation, with or without gas intraocular tamponade. ${ }^{1-4}$ However, in spite of maximum photocoagulation retinal ischaemia may continue to provide a stimulus to the proliferative process involving the vitreous base, the anterior segment and occasionally leading to rubeosis. ${ }^{11}$ In such a selected group of eyes, tamponade with silicone oil is at times used at this institution. The ultimate goal of such surgery should be removal of the silicone oil However this was not possible because of the presence of inferior traction detachments and retinal break formation.

The phenomenon of reproliferation of fibrocellular tissue, characteristically involving the inferior retinal periphery can be explained by the presence of silicone oil in such eyes. The silicone oil globule is buoyant and as it is impossible to fill $100 \%$ of the vitreous cavity with silicone oil the inferior peripheral retina remains in contact with aqueous. Theoretically, growth factors become effectively 'compartmentalised' by the silicone oil bubble. ${ }^{10,12}$ Inferior peripheral retinal detachment may thus occur.

The peripheral retina is thinner than posterior retina. In diabetic eyes where severe ischaemia and extensive panretinal photocoagulation make the peripheral retina atrophic it is therefore more liable to develop breaks in the presence of tangential traction. In all of our cases the posterior retina remains attached.

As with other extramacular traction retinal detachments in diabetic eyes we decided upon a conservative approach to observe the behaviour of these localised detachments. Charles has recommended immediate reoperation in diabetic eyes showing signs of anterior vitreous fibrovascular proliferation following vitrectomy. ${ }^{2}$ This would seem reasonable, as such eyes may develop severe haemorrhage and in the presence of long acting gases, be very likely to develop severe anterior proliferative vitreoretinopathy. ${ }^{13}$

We would wish to comment upon the apparently benign behaviour of peripheral reproliferation in the presence of silicone oil and emphasise the slow course of events leading to stabilisation of the retina, allowing the posterior ret- ina to remain attached with improvement of central vision. Although to date we have not removed the silicone oil from these eyes, we can speculate that it would be safe to do so after completion of the retinotomy if indicated.

We thank Mr. T. Tarrant for kindly providing Figs. 1-4.

Key words: Diabetic retinopathy, Retinotomy, Silicone oil, Tension tears, Vitrectomy.

\section{REFERENCES}

1. Blankenship G: Pars plana vitrectomy for diabetic retinopathy: A report of eight years experience. Mod Prob Ophthalmol 1979, 20: 376-86.

2. Charles S: Vitreous microsurgery, 2nd ed. Baltimore: Williams \& Wilkins, 1987.

3. McLeod D, James CR: Viscodelamination at the vitreoretinal juncture in severe diabetic eye disease. $\mathrm{Br} J$ Ophthalmol 1988; 72: 413-9.

4. Williams DF, Williams GA, Hartz A, Meiler, WF, Abrams GA, Aaberg TM: Results of vitrectomy for diabetic traction retinal detachment using the en bloc excision technique. Ophthalmology 1989, 96: 752-8.

5. McLeod D: Use of silicone oil in diabetic vitrectomy. Internat Ophthalmol 1985, 8: 104-5.

6. McLeod D: Silicone-oil injection during closed microsurgery for diabetic retinal detachment. Von Graefe's Arch Clin Exp Ophthalmol 1986, 224: 55-9.

7. Leaver PK, Grey RHB, Garner A: Silicone oil injection in the management of massive preretinal retraction. II. Late complications in 93 cases. $\mathrm{Br} J$ Ophthalmol 1979, 63: 361-7.

8. Haut J, Ullern, Chermet M, Van Effenterre G: Complications of intraocular injections of silicone combined with vitrectomy. Ophthalmologica 1980, 180: 29-35.

9. Leaver PK, Grey RHB, Garner A: Complications following silicone oil injection. In Streiff ED, ed. Modern problems in ophthalmology. Basel: Karger, 1979, 20: 290-4.

10. Boulton M, McLeod D, Garner A: Vasoproliferative retinopathies: clinical, morphogenetic and modulatory aspects. Eye 1988, 2: s124-39.

11. Lewis H, Abrams GW, Williams GA: Anterior hyaloidal fibrovascular proliferation after diabetic vitrectomy. $A m \mathrm{~J}$ Ophthalmol 1987, 104: 607-13.

12. Yeo JH, Glaser BM, Michels RG: Silicone oil in the treatment of complicated retinal detachments. Ophthalmology 1987, 94: 1109-13.

13. Chang S, Lincoff HA, Coleman D, et al.: Perfluorocarbon gases in vitreous surgery. Ophthalmology 1985, 92: 651-6. 